\title{
Loco-regional recurrence trend and prognosis in young women with breast cancer according to molecular subtypes: analysis of 1099 cases
}

Yang Li', Su Lu', Yuhan Zhang ${ }^{2}$, Shuaibing Wang ${ }^{3}$ and Hong Liu ${ }^{1 *}$ (D)

\begin{abstract}
Background: The number of young patients diagnosed with breast cancer is on the rise. We studied the rate trend of local recurrence (LR) and regional recurrence (RR) in young breast cancer (YBC) patients and outcomes among these patients based on molecular subtypes.

Methods: A retrospective cohort study was conducted based on data from Tianjin Medical University Cancer Institute and Hospital for patients $\leq 35$ years of age with pathologically confirmed primary invasive breast cancer surgically treated between 2006 and 2014. Patients were categorized according to molecular subtypes on the basis of hormone receptor (HR) and human epidermal growth factor receptor 2 (HER2) status. The 5-year rates for LR, RR, and distant metastases (DM) were estimated by Kaplan-Meir statistics. Nelson-Aalen cumulative-hazard plots were used to describe local recurrence- and distant metastasis-free intervals.
\end{abstract}

Results: We identified 25,284 patients with a median follow-up of 82 months, of whom 1099 (4.3\%) were YBC patients $\leq 35$ years of age. The overall 5-year LR, RR, and DM rates in YBC patients were $6.7 \%, 5.1 \%$, and 16.6\%, respectively. The $L R$ and RR rates demonstrated a decreasing trend over time $(P=0.028$ and $P=0.015$, respectively). We found that early-stage breast cancer and less lymph node metastases increased over time $(P=0.004$ and $P=$ 0.007, respectively). Patients with HR-/HER2+ status had a significantly higher LR (HR 20.4; 95\% Cl, 11.8-35.4) and DM (HR 37.2; 95\% Cl, 24.6-56.3) at 10 years. Breast-conserving surgery (BCS) or mastectomy did not influence rates of LR and RR. In the overall population, the 5-year survival of YBC patients exceeded $90 \%$.

Conclusions: The rates of $L R$ and RR with YBC patients demonstrated a downward trend and the proportion of early-stage breast cancer increased between 2006 and 2014. We report the highest LR rates in this young population were associated with HR-/HER2+ tumors.

Keywords: Young breast cancer, Local recurrence, Regional recurrence, Distant metastases, Molecular subtype, Overall survival

\footnotetext{
* Correspondence: liuhongzhang0101@163.com

${ }^{1}$ The Second Surgical Department of Breast Cancer, Tianjin Medical University Cancer Institute and Hospital, National Clinical Research Center for Cancer \& Key Laboratory of Cancer Prevention and Therapy, Tianjin's Clinical Research Center for Cancer, Tianjin 300060, China

Full list of author information is available at the end of the article
}

(C) The Author(s). 2021 Open Access This article is licensed under a Creative Commons Attribution 4.0 International License, which permits use, sharing, adaptation, distribution and reproduction in any medium or format, as long as you give appropriate credit to the original author(s) and the source, provide a link to the Creative Commons licence, and indicate if changes were made. The images or other third party material in this article are included in the article's Creative Commons licence, unless indicated otherwise in a credit line to the material. If material is not included in the article's Creative Commons licence and your intended use is not permitted by statutory regulation or exceeds the permitted use, you will need to obtain permission directly from the copyright holder. To view a copy of this licence, visit http://creativecommons.org/licenses/by/4.0/ The Creative Commons Public Domain Dedication waiver (http://creativecommons.org/publicdomain/zero/1.0/) applies to the data made available in this article, unless otherwise stated in a credit line to the data. 


\section{Introduction}

It has been estimated that $4 \%$ of females $<40$ years of age were diagnosed with breast cancer in the United States in 2017 and breast cancer is the leading cause of cancer deaths among women 20-59 years of age [1, 2]. Prior studies have revealed that young age is a known risk prognostic factor for breast cancer patients [3-8]. This finding is reflected by larger tumors, higher grade, advanced stage, more lymph node metastases, a higher prevalence of human epidermal growth factor receptor (HER)2 over-expression, and estrogen receptor (ER) negativity in young women with breast cancer $[4,5,7]$. With respect to detrimental gene expression, Azim et al. reported higher expression of gene signatures related to proliferation, stem cells, and endocrine resistance in tumors associated with young age [9]. In addition, higher expression of epithelial growth factor receptor (EGFR) mRNA, which BRCA1/2-associated breast tumors overexpress [10], is a significant predictor of poor prognosis in young women [5].

The relative risk of loco-regional recurrence (LRR) increases by $7 \%$ for every year of decrease in age [11]. Previous research showed that young women with breast cancer who undergo breast-conserving surgery (BCS) have higher rates of developing LRR compared with women who undergo mastectomy, but the overall survival (OS) is not affected $[12,13]$. Despite higher rates of LRR in young patients, several studies had shown a declining trend in the LRR rate over the past two decades [14-16]. In addition, there has been a significant decline in the occurrence of distant metastases (DM) $[17,18]$ and increase in the OS over the last years in YBC patients owing to the evolution of improved adjuvant systemic treatment and raising consciousness of physical examination $[19,20]$.

Breast cancer arising in young women is more likely to develop into more aggressive tumor subtypes, including a greater proportion of triple-negative and HER2 over-expressing subtypes [5, 6, 8]. Accumulating evidence has demonstrated a strong relationship between molecular subtypes and prognosis in YBC patients [21-32]. A number of reports have shown a worse OS rate in young women with luminal B breast cancer [27, 28, 31, 32], whereas other research has suggested that triple-negative and HER2 overexpressing tumors are strong predictors of disease recurrence [29, 30, 33]. Therefore, larger, well-designed prospective clinical studies are needed to explore this relationship.

The trend in LRR rates in YBC patients in recent years has not been established. We therefore evaluated the trend in LRR and determine the impact of molecular subtypes on LRR and OS in young women diagnosed with breast cancer.

\section{Methods}

\section{Patients}

This was a retrospective study that included breast cancer patients $\leq 35$ years of age at Tianjin Medical University Cancer Institute and Hospital from January 2006 to December 2014. There were 25,284 patients diagnosed with breast cancer in our hospital during the 9-year period, of whom 1307 were $\leq 35$ years of age. Patients $\leq 35$ years of age with pathologically confirmed primary invasive breast cancer and underwent surgery from 2006 to 2014 were selected for our study. Subjects with non-invasive cancer (54 cases), including ductal carcinoma in situ (16 cases), primary metastatic breast cancer (20 cases), and primary bilateral breast cancer (42 cases), were excluded. We also excluded patients who did not have electronic medical records in our institution and who could not be contacted by telephone or mail to confirm survival status (92 cases). A total of 1099 young women with breast cancer met the inclusion criteria for our study.

\section{Clinicopathological information of patients}

We collected the following patient demographics: age; family history of breast cancer; reproductive history; and breastfeeding history. The tumor characteristics included tumor size, stage, lymph node status, histologic grade, and pathologic type. We classified cancer into five molecular subtypes according to hormone receptor (HR) and HER2 status, as follows: HR-positive/Her2-negative; HR-positive/Her2-positive; HR-negative/Her2-positive; HR-negative/Her2-negative; and unknown. Molecular subtype was defined by immunohistochemical staining features of HR (estrogen receptor [ER] and/or progesterone receptor $[\mathrm{PR}]$ ) and HER2. Categorization based on staining features was as follows: ER and PR staining < $1 \%$ was defined as negative; ER and/ or PR staining $\geq 1 \%$ was defined as positive [34]; HER2 0/1 was defined as negative; and HER2 2+ was defined as negative or positive by fluorescence in situ hybridization (FISH) and positive by HER2 $3+$. Information regarding adjuvant chemotherapy, radiotherapy, hormonal therapy, ovarian function suppression, and trastuzumab therapy were obtained from the hospital and follow-up records.

\section{Variable definitions}

The follow-up data for LR, RR, DM, and OS were abstracted from the electronic medical records, paper medical documents, telephone, and mail. For patients at the time of contact had died, available family members provided the requested information. Follow-up started on the day of surgery to the date of any type of recurrence, death, the last contact according to the medical record, or in-person contact. LR was defined as recurrence of ipsilateral breast cancer after BCS or chest wall recurrence 
after mastectomy. RR referred to the occurrence of tumor in the ipsilateral regional lymph nodes, including the axillary, infra- or supra-clavicular or internal mammary lymph nodes. DM was defined as recurrence beyond LR and RR. We defined OS as the time from surgery to death from any cause or last follow-up. The local recurrence-free interval (LRFI) was defined as the interval from surgery to local recurrence or the date of last follow-up.

\section{Statistical analysis}

Descriptive statistics were performed to examine the demographic characteristics of young patients surgically treated between 1 January 2006 and 31 December 2014. The percentage of clinicopathologic and therapeutic regimen among YBC patients were compared for the different molecular subtypes using a chi-square test. Moreover, tumor characteristics for all YBC patients according to the time of diagnosis were assessed over time.

We used Kaplan-Meier survival estimates to calculate overall 5-year LR, RR, and DM rates for the young patients with breast cancer and the trends of LR, RR and DM over time were assessed by using linear regression analysis. Moreover, LR, RR, and DM rates of YBC patients treated between 2006 and 2014 according to various molecular subtypes were calculated. We performed univariate and multivariate Cox proportional hazard model to examine the influence of different variables on LR, RR, and DM. Hazard ratios and the associated 95\% confidence intervals (CIs) were obtained based on Cox regression analysis. The OS was summarized by KaplanMeier survival curves according to tumor subtypes and compared using log-rank test univariate analyses. Nelson-Aalen cumulative-hazard plots were used to describe the LRFI and distant metastases-free interval (DMFI). Subsequently, 5- and 10-year estimates of LRFI, regional recurrence-free interval (RRFI), DMFI, and OS according to various molecular subtypes were calculated using Kaplan-Meier survival analysis.

$P$ values $<0.05$ were considered statistically significant and all tests were two-tailed. Analyses were performed using SPSS 22.0 and STATA software 14.1.

\section{Results}

\section{Patient characteristics}

A total of 1099 YBC patients who were surgically treated were enrolled in our study from 2006 to 2014. This cohort accounted for $4.3 \%$ of the total population of patients who were diagnosed with breast cancer in our hospital during the 9-year period ( $n=25,284)$. The median follow-up time was 82 months. The demographic characteristics of the YBC patients are shown in Table 1. Seventy-five percent of the patients had early-stage breast cancer (stages I and II). Among the patients,
$54.0 \%, 10.9 \%, 6.8 \%$, and $18.3 \%$ of patients were $\mathrm{HR}+\mathrm{l}$ HRE2-, HR+/Her2+, HR-/Her2+, and HR-/HER2subtypes, respectively. The baseline clinicopathologic and treatment characteristics differed by tumor subtype, as shown in Table 2. Patients with HER2 2+ status who were not subsequently detected by FISH were classified as unknown subtype. HR+/HER2- tumors tended to be smaller in size $(P=0.007)$, lower stage $(P<0.001)$, and lower histologic grade $(P<0.001)$ compared with the other subtypes. Patients with HR-/HER2+ status were likely to have larger tumors $(P=0.007)$ and patients with HER2-negative breast cancer presented with fewer lymph node metastases, while HER2-positive tumors tended to have $>9$ lymph node metastases $(P<0.001)$.

As for treatment, patients with HER2-negative tumors generally underwent BCS $(P=0.002)$ compared with HER2-positive tumors. The percentage of patients with HR-/HER2+ and HR-/HER2- statuses receiving neoadjuvant chemotherapy $(P=0.003)$ and radiotherapy treatment $(P<0.001)$ was higher than other subtypes. No statistically significant difference was observed in chemotherapy among patients with different molecular subtypes. There were 194 women with HER2-positive status and 83 patients received trastuzumab therapy, of whom 20 relapsed after surgery in the current study.

In addition, we studied the distribution of tumor characteristics and treatment for all YBC patients over time shown in Table 3. Tumor size, histologic grade, pathologic type, and type of surgery did not vary significantly between 2006 and 2014. Of note, there were distinct proportional shifts of stage and lymph node metastases over time $(P=0.004$ and $P=0.007$, respectively). The proportion of $\mathrm{N} 1$ increased $(P=0.016$ using linear regression analyses), while $\mathrm{N} 2$ and $\mathrm{N} 3$ showed a declining trend, although no significant difference was detected using linear regression analyses. The percentage of patients with stage II breast cancer was higher and the percentage of patients with breast cancer stage III trended down over time. The proportion of patients receiving neoadjuvant chemotherapy $(P=0.375)$ and radiotherapy $(P=0.512)$ did not increase significantly over time (Fig. 1). There was a significant proportional shift of receiving chemotherapy during the study period: the proportion of patients treated with anthracyclineand taxane-based regimen increased $(P=0.003)$. The proportion of patients receiving trastuzumab increased over time $(P<0.001)$.

\section{Recurrence rates}

LR occurred in 83 patients in this study. There were 11 cases receiving surgical resection only, 35 cases using surgery and chemotherapy, 20 patients receiving chemotherapy only, 12 cases taking surgery and radiotherapy and chemotherapy, and the remaining 5 cases were 
Table 1 Demographic characteristics of young patients surgically treated between January 1, 2006, and December 31, $2014(n=1099)$

\begin{tabular}{|c|c|}
\hline Characteristics & No. of pat \\
\hline \multicolumn{2}{|l|}{ BC family history } \\
\hline Yes & $113(10.3)$ \\
\hline No & $969(88.2)$ \\
\hline Unknown & $17(1.5)$ \\
\hline \multicolumn{2}{|l|}{ Reproductive history* } \\
\hline Yes & $827(75.3)$ \\
\hline No & $257(23.4)$ \\
\hline Unknown & $15(1.4)$ \\
\hline \multicolumn{2}{|l|}{ Breastfeeding history } \\
\hline Yes & $745(67.8)$ \\
\hline No & $337(30.7)$ \\
\hline Unknown & $17(1.5)$ \\
\hline \multicolumn{2}{|l|}{ Tumor size } \\
\hline $\mathrm{T} 1$ & $420(38.2)$ \\
\hline $\mathrm{T} 2$ & $508(46.2)$ \\
\hline T3 & $88(8.0)$ \\
\hline T4 & $16(1.5)$ \\
\hline Tx & $67(6.1)$ \\
\hline \multicolumn{2}{|l|}{ Stage } \\
\hline । & $282(25.7)$ \\
\hline$\| \mathrm{a}$ & $383(34.8)$ \\
\hline$\| \mathrm{b}$ & $159(14.5)$ \\
\hline III a & $126(11.5)$ \\
\hline III b & $8(0.7)$ \\
\hline III C & $97(8.8)$ \\
\hline Unknown & $44(4.0)$ \\
\hline \multicolumn{2}{|l|}{ Lymph node metastasis } \\
\hline NO & $585(53.2)$ \\
\hline $\mathrm{N} 1$ & $276(25.1)$ \\
\hline N2 & $124(11.3)$ \\
\hline N3 & $94(8.6)$ \\
\hline Unknown & $20(1.8)$ \\
\hline \multicolumn{2}{|l|}{ Histological grade } \\
\hline Well differentiated & $35(3.2)$ \\
\hline Moderately differentiated & $594(54.0)$ \\
\hline Poorly differentiated & $163(14.8)$ \\
\hline Unknown & 307 (27.9) \\
\hline \multicolumn{2}{|l|}{ Pathological type } \\
\hline Invasive ductal carcinoma & $999(90.9)$ \\
\hline Invasive lobular carcinoma & $10(0.9)$ \\
\hline Others & $90(8.2)$ \\
\hline
\end{tabular}

Table 1 Demographic characteristics of young patients surgically treated between January 1, 2006, and December 31, $2014(n=1099)$ (Continued)

\begin{tabular}{|c|c|}
\hline Characteristics & No. of patients (\%) \\
\hline \multicolumn{2}{|l|}{ Final surgery } \\
\hline Breast-conserving surgery & $257(23.4)$ \\
\hline Mastectomy & $842(76.6)$ \\
\hline \multicolumn{2}{|l|}{ Biomarker subtype } \\
\hline $\mathrm{HR}+/ \mathrm{HER} 2-$ & $594(54.0)$ \\
\hline $\mathrm{HR}+/ \mathrm{HER} 2+$ & $120(10.9)$ \\
\hline HR-/HER2+ & $75(6.8)$ \\
\hline HR-/HER2- & $201(18.3)$ \\
\hline Unknown & $109(9.9)$ \\
\hline \multicolumn{2}{|l|}{ Neoadjuvant chemotherapy } \\
\hline Yes & $176(16.0)$ \\
\hline No & $923(84.0)$ \\
\hline \multicolumn{2}{|l|}{ Adjuvant chemotherapy regimens } \\
\hline Anthracycline-based & $140(12.7)$ \\
\hline Anthracycline- and taxane-based & $884(80.4)$ \\
\hline Unknown & $67(6.1)$ \\
\hline None & $8(0.7)$ \\
\hline \multicolumn{2}{|l|}{ Radiotherapy } \\
\hline Yes & $557(50.7)$ \\
\hline No & $453(41.2)$ \\
\hline Unknown & $89(8.1)$ \\
\hline \multicolumn{2}{|l|}{ Endocrine therapy } \\
\hline Yes & $589(53.6)$ \\
\hline No & $316(28.8)$ \\
\hline Unknown & $194(17.7)$ \\
\hline \multicolumn{2}{|l|}{ Ovarian function suppression } \\
\hline Yes & $187(17.0)$ \\
\hline No & $545(49.6)$ \\
\hline Unknown & $367(33.4)$ \\
\hline \multicolumn{2}{|l|}{ Trastuzumab treatment } \\
\hline Yes & $85(7.7)$ \\
\hline No & $836(76.1)$ \\
\hline Unknown & $178(16.2)$ \\
\hline
\end{tabular}

$B C$, breast cancer; $H R+$, hormone receptor positive, $H R$ - hormone receptor negative, HER2+ human epidermal growth factor 2 positive, HER2- human epidermal growth factor 2 negative

*Reproductive history: Yes means they had children

unknown. A total of 211 patients occurred DM. The treatment of 34 patients after recurrence was unknown and the remaining 177 patients were treated with chemotherapy as the main combination therapy. The overall 5-year LR, RR, and DM rates in YBC patients were $6.7 \%, 5.1 \%$, and $16.6 \%$, respectively. We used linear regression analyses to evaluate the time trend of 
Table 2 Baseline demographic characteristics of all young patients according to various molecular subtypes $(n=1099)$

\begin{tabular}{|c|c|c|c|c|c|c|}
\hline Characteristics & $\begin{array}{l}\text { HR+/HER2- } \\
(n=594)\end{array}$ & $\begin{array}{l}\text { HR+/HER2+ } \\
(n=120)\end{array}$ & $\begin{array}{l}\text { HR-/HER2+ } \\
(n=75)\end{array}$ & $\begin{array}{l}\text { HR-/HER2- } \\
(n=201)\end{array}$ & $\begin{array}{l}\text { Unknown } \\
(n=109)\end{array}$ & $P$ \\
\hline BC family history & & & & & & 0.023 \\
\hline YES & $62(10.4)$ & $16(13.3)$ & $5(6.7)$ & $21(10.4)$ & $9(8.3)$ & \\
\hline NO & $524(88.2)$ & $104(86.7)$ & $68(90.7)$ & $179(89.1)$ & $94(86.2)$ & \\
\hline Unknown & $8(1.3)$ & $0(0)$ & $2(2.7)$ & $1(0.5)$ & $6(5.5)$ & \\
\hline Reproductive history & & & & & & 0.003 \\
\hline YES & $451(75.9)$ & $92(76.7)$ & $61(81.3)$ & $146(72.6)$ & 77 (70.6) & \\
\hline NO & $136(22.9)$ & $28(23.3)$ & $12(16.0)$ & $55(27.4)$ & $26(23.9)$ & \\
\hline Unknown & $7(1.2)$ & $0(0)$ & $2(2.7)$ & $0(0)$ & $6(5.5)$ & \\
\hline Breastfeeding history & & & & & & $<0.001$ \\
\hline YES & $410(69.0)$ & 79 (65.8) & $59(78.7)$ & $128(63.7)$ & $69(63.3)$ & \\
\hline NO & $177(29.8)$ & $41(34.2)$ & $14(18.7)$ & $72(35.8)$ & $33(30.3)$ & \\
\hline Unknown & $7(1.2)$ & $0(0)$ & $2(2.7)$ & $1(0.5)$ & $7(6.4)$ & \\
\hline Tumor size & & & & & & 0.007 \\
\hline $\mathrm{T} 1$ & $250(42.1)$ & $39(32.5)$ & $17(22.7)$ & $69(34.3)$ & $45(41.3)$ & \\
\hline $\mathrm{T} 2$ & $261(43.9)$ & $61(50.8)$ & $40(53.3)$ & $103(51.2)$ & $43(39.4)$ & \\
\hline T3 & $45(7.6)$ & $10(8.3)$ & $8(10.7)$ & $12(6.0)$ & $13(11.9)$ & \\
\hline T4 & $3(0.5)$ & $5(4.2)$ & $3(4.0)$ & $4(2.0)$ & $1(0.9)$ & \\
\hline Tx & $35(5.9)$ & $5(4.2)$ & $7(9.3)$ & $13(6.5)$ & $7(6.4)$ & \\
\hline Stage & & & & & & $<0.001$ \\
\hline I & $166(27.9)$ & $27(22.5)$ & $8(10.7)$ & 48 (23.9) & $33(30.3)$ & \\
\hline$\| \mathrm{a}$ & $199(33.5)$ & $40(33.3)$ & $26(34.7)$ & $90(44.8)$ & $28(25.7)$ & \\
\hline$\| \mathrm{b}$ & 85 (14.3) & $16(13.3)$ & $13(17.3)$ & $28(13.9)$ & 17 (15.6) & \\
\hline III a & $72(12.1)$ & $15(12.5)$ & $12(16.0)$ & $17(8.5)$ & $10(9.2)$ & \\
\hline III b & $2(0.3)$ & $1(0.8)$ & $2(2.7)$ & $3(1.5)$ & $0(0)$ & \\
\hline III C & $48(8.1)$ & $17(14.2)$ & $12(6.0)$ & $4(2.0)$ & $16(14.7)$ & \\
\hline Unknown & $22(3.7)$ & $4(3.3)$ & $2(2.7)$ & $11(5.5)$ & $5(4.6)$ & \\
\hline LN metastasis & & & & & & $<0.001$ \\
\hline NO & $312(52.5)$ & $55(45.8)$ & $36(48.0)$ & $134(66.7)$ & $48(44.0)$ & \\
\hline $\mathrm{N} 1$ & $161(27.1)$ & $30(25.0)$ & 14 (18.7) & 46 (22.9) & $25(22.9)$ & \\
\hline $\mathrm{N} 2$ & $70(11.8)$ & $16(13.3)$ & $13(17.2)$ & $16(8.0)$ & $9(8.3)$ & \\
\hline N3 & $46(7.7)$ & $17(14.2)$ & $12(16.0)$ & $2(1.0)$ & $17(15.6)$ & \\
\hline Unknown & $5(0.8)$ & $2(1.7)$ & $0(0)$ & $3(1.5)$ & $10(9.2)$ & \\
\hline Histological grade & & & & & & $<0.001$ \\
\hline Well & $29(4.9)$ & $0(0)$ & $1(1.3)$ & $2(1.0)$ & $3(2.8)$ & \\
\hline Moderately & $371(62.5)$ & $67(55.8)$ & $36(48.0)$ & 78 (38.8) & $42(38.5)$ & \\
\hline Poorly & $50(8.4)$ & $25(20.8)$ & $16(21.3)$ & $52(25.9)$ & $20(18.3)$ & \\
\hline Unknown & $144(24.2)$ & $28(23.3)$ & $22(29.3)$ & 69 (34.3) & $44(40.4)$ & \\
\hline Pathological type & & & & & & 0.906 \\
\hline IDC & $534(89.9)$ & $110(91.7)$ & $71(94.7)$ & $183(91.0)$ & $101(92.7)$ & \\
\hline $\mathrm{IBC}$ & $7(1.2)$ & $1(0.8)$ & $0(0)$ & $1(0.5)$ & $1(0.9)$ & \\
\hline Others & $53(8.9)$ & $9(7.5)$ & $4((5.3)$ & $17(8.5)$ & $7(6.4)$ & \\
\hline Final surgery & & & & & & 0.002 \\
\hline BCS & $143(24.1)$ & 19 (15.8) & 14 (18.7) & $64(31.8)$ & 17 (15.6) & \\
\hline
\end{tabular}


Table 2 Baseline demographic characteristics of all young patients according to various molecular subtypes $(n=1099)$ (Continued)

\begin{tabular}{|c|c|c|c|c|c|c|}
\hline Characteristics & $\begin{array}{l}\text { HR+/HER2- } \\
(n=594)\end{array}$ & $\begin{array}{l}\text { HR+/HER2+ } \\
(n=120)\end{array}$ & $\begin{array}{l}\text { HR-/HER2+ } \\
(n=75)\end{array}$ & $\begin{array}{l}\text { HR-/HER2- } \\
(n=201)\end{array}$ & $\begin{array}{l}\text { Unknown } \\
(n=109)\end{array}$ & $P$ \\
\hline Mastectomy & $451(75.9)$ & $101(84.2)$ & $61(81.3)$ & $137(68.2)$ & $92(84.4)$ & \\
\hline NACT & & & & & & 0.003 \\
\hline YES & $80(13.5)$ & 19 (15.8) & $23(30.7)$ & $33(16.4)$ & $21(19.3)$ & \\
\hline NO & $514(86.5)$ & $101(84.2)$ & $52(69.3)$ & $168(83.6)$ & $88(80.7)$ & \\
\hline $\mathrm{ACT}$ & & & & & & 0.046 \\
\hline A-based & $72(12.1)$ & $17(14.2)$ & $12(16.0)$ & $24(11.9)$ & 15 (13.8) & \\
\hline A- and T-based & $490((82.5)$ & 97 (80.8) & $58(77.3)$ & $162(80.6)$ & 77 (70.6) & \\
\hline Unknown & $28(4.7)$ & $6(5.0)$ & $5(6.7)$ & $12(6.0)$ & $16(14.7)$ & \\
\hline None & $4(0.7)$ & $0(0)$ & $0(0)$ & $3(1.5)$ & $1(0.9)$ & \\
\hline Radiotherapy & & & & & & $<0.001$ \\
\hline YES & $304(51.2)$ & $59(49.2)$ & $46(61.3)$ & $105(52.2)$ & $43(39.4)$ & \\
\hline NO & $254(42.8)$ & $52(43.3)$ & $24(32.0)$ & $85(42.3)$ & $38(34.9)$ & \\
\hline Unknown & $36(6.1)$ & $9(7.5)$ & $5(6.7)$ & $11(5.5)$ & $28(25.7)$ & \\
\hline ET & & & & & & $<0.001$ \\
\hline YES & 467 (78.6) & $89(74.2)$ & $0(0)$ & $0(0)$ & $33(30.3)$ & \\
\hline NO & $27(4.5)$ & $8(6.7)$ & $70(93.3)$ & $194(96.5)$ & $17(15.6)$ & \\
\hline Unknown & $100(16.8)$ & $23(19.2)$ & $5(6.7)$ & $7(3.5)$ & $59(54.1)$ & \\
\hline OFS & & & & & & $<0.001$ \\
\hline YES & $147(24.7)$ & $34(28.3)$ & $0(0)$ & $0(0)$ & $6(5.5)$ & \\
\hline NO & $215(36.2)$ & $46(38.3)$ & $67(89.3)$ & $189(94.0)$ & $28(25.7)$ & \\
\hline Unknown & $232(39.1)$ & $40(33.3)$ & $8(10.7)$ & $12(6.0)$ & $75(68.8)$ & \\
\hline$\pi$ & & & & & & $<0.001$ \\
\hline YES & $2(0.3)$ & $51(42.5)$ & $32(42.7)$ & $0(0)$ & $0(0)$ & \\
\hline NO & $553(93.1)$ & $41(34.2)$ & $23(30.7)$ & $195(97.0)$ & $24(22.0)$ & \\
\hline Unknown & $39(6.6)$ & $28(23.3)$ & $20(26.7)$ & $6(3.0)$ & 85 (78.0) & \\
\hline
\end{tabular}

All data are given as No. of patients (\%). None represents no chemotherapy has been adopted

$L N$, lymph node; IDC, invasive ductal carcinoma; IBC, invasive lobular carcinoma; $N A C T$, neoadjuvant chemotherapy; $B C S$, breast conserving surgery; $A$-based,

anthracycline-based; $A$ - and T-based, anthracycline- and taxane-based; OFS, ovarian function suppression; $E T$, endocrine therapy; $T T$, trastuzumab therapy

recurrence rates over the 9-year period. The LR and RR rates demonstrated a decreasing trend over time $(P=$ 0.028 and $P=0.015$, respectively). The DM rate also declined, although the difference was not statistically significant $(P=0.228)$, as is shown in Table 4 .

There were statistically significant differences in the LR and DM rates in patients with various tumor subtypes $(P=0.002$ and $P=0.003$, respectively; Fig. 2$)$. Patients with HR-/HER2+ tumors had the highest recurrence rate compared with the other subtypes (LR: 17.3\%, RR: 9.3\%, and DM: 30.7\%). Patients with $\mathrm{HR+1}$ HER2 - status displayed the lowest LR rate (5.6\%), whereas the triple-negative subtype showed the lowest DM rate (13.4\%). We used univariate and multivariate Cox proportional hazard models to analyze the prognostic factors, as shown in Table 5 and Table 6. Patients with larger tumors and more lymph node metastases had increased HR in multivariate analyses for LRR $(P<$ $0.01)$. The type of surgery did not influence the risk of
LR and RR. LR and RR were $17.0 \%$ and $5.4 \%$ after BCS versus $7.9 \%$ and $8.3 \%$ after a mastectomy $(P=0.124, P=$ 0.296 , respective). In addition, a total of 36 patients underwent sentinel lymph node biopsies (SLNB) in our study, including 2 patients with distant metastases (one liver metastases and the other bone and ovary metastases, respectively), 1 with regional lymph node metastases, and 1 with local recurrence and brain metastases.

\section{Survival outcomes}

HR-/HER2+ patients had the worse OS compared with patients with the other subtypes $(P<0.001$; Fig. 3$)$. Table 7 lists the relapse and OS for patients with various molecular subtypes. HR-/HER2+ patients had the worst LRFI, RRFI, DMFI, and OS compared with patients with the other subtypes. The median follow-up time was 82 months (range, 5-156 months). In the overall population, the 5-year survival of young patients with breast cancer surgically treated in our institution exceeded 
Table 3 Tumor characteristics for all young breast cancer patients according to the time of diagnosis $(n=1099)$

\begin{tabular}{|c|c|c|c|c|c|c|c|c|c|c|}
\hline & $\begin{array}{l}2006 \\
n=55\end{array}$ & $\begin{array}{l}2007 \\
n=73\end{array}$ & $\begin{array}{l}2008 \\
n=96\end{array}$ & $\begin{array}{l}2009 \\
n=93\end{array}$ & $\begin{array}{l}2010 \\
n=132\end{array}$ & $\begin{array}{l}2011 \\
n=154\end{array}$ & $\begin{array}{l}2012 \\
n=147\end{array}$ & $\begin{array}{l}2013 \\
n=166\end{array}$ & $\begin{array}{l}2014 \\
n=183\end{array}$ & $P$ \\
\hline \multicolumn{11}{|l|}{ Tumor size } \\
\hline $\mathrm{T} 1$ & $21(38)$ & $27(37)$ & $39(41)$ & $37(40)$ & $45(34)$ & $64(42)$ & $49(33)$ & $62(37)$ & $76(42)$ & 0.539 \\
\hline $\mathrm{T} 2$ & $30(55)$ & $34(47)$ & $43(45)$ & $42(45)$ & $61(46)$ & $65(42)$ & $75(51)$ & $82(49)$ & $76(42)$ & \\
\hline T3 & $2(4)$ & $3(4)$ & $5(5)$ & $5(5)$ & $16(12)$ & $13(8)$ & $15(10)$ & $12(7)$ & $17(9)$ & \\
\hline $\mathrm{T} 4$ & $1(2)$ & $2(3)$ & $3(3)$ & $0(0)$ & $1(1)$ & $1(1)$ & $4(3)$ & $2(1)$ & $2(1)$ & \\
\hline$T x$ & $1(2)$ & $7(10)$ & $6(6)$ & $9(10)$ & $9(7)$ & $11(7)$ & $4(3)$ & $8(5)$ & $12(7)$ & \\
\hline Stage & & & & & & & & & & 0.004 \\
\hline 1 & $11(20)$ & $21(29)$ & $27(28)$ & $24(26)$ & $30(23)$ & $40(26)$ & $35(24)$ & $45(27)$ & $49(27)$ & \\
\hline$\| \mathrm{a}$ & $24(44)$ & $20(27)$ & 37 (39) & $36(39)$ & 41 (31) & $47(31)$ & $56(38)$ & $51(31)$ & 71 (39) & \\
\hline$\| b$ & $4(7)$ & $11(15)$ & $9(9)$ & $13(14)$ & $17(13)$ & $19(12)$ & 27 (18) & $36(22)$ & $23(13)$ & \\
\hline III a & $13(23)$ & $7(10)$ & $8(8)$ & $8(9)$ & $22(17)$ & $20(13)$ & $13(9)$ & $14(8)$ & $21(12)$ & \\
\hline III b & $0(0)$ & $2(3)$ & $0(0)$ & $0(0)$ & $0(0)$ & $0(0)$ & $2(1)$ & $2(1)$ & $2(1)$ & \\
\hline III C & $3(6)$ & $5(7)$ & $14(15)$ & $4(4)$ & $14(11)$ & $21(14)$ & $12(8)$ & $13(8)$ & $11(6)$ & \\
\hline Unknown & $0(0)$ & $7(10)$ & $1(1)$ & $8(9)$ & $8(6)$ & $7(5)$ & $2(1)$ & $5(3)$ & $6(3)$ & \\
\hline LNM & & & & & & & & & & 0.007 \\
\hline No & $30(55)$ & $42(58)$ & $56(58)$ & $52(56)$ & $67(51)$ & $73(47)$ & $80(54)$ & $83(50)$ & $102(56)$ & \\
\hline N1 & $8(15)$ & $15(21)$ & $19(20)$ & $26(28)$ & $30(23)$ & $38(25)$ & $41(28)$ & $54(33)$ & $45(25)$ & \\
\hline N2 & $13(24)$ & $11(15)$ & $6(6)$ & $10(11)$ & 19 (14) & $20(13)$ & $12(8)$ & $15(9)$ & $18(10)$ & \\
\hline N3 & $4(7)$ & $5(7)$ & $14(15)$ & $5(5)$ & $14(11)$ & $21(14)$ & $11(8)$ & $11(7)$ & $9(5)$ & \\
\hline Unknown & $0(0)$ & $0(0)$ & $1(1)$ & $0(0)$ & $2(2)$ & $2(1)$ & $3(2)$ & $3(2)$ & $9(4.9)$ & \\
\hline $\mathrm{HG}$ & & & & & & & & & & 0.415 \\
\hline Well & $4(7)$ & $2(3)$ & $6(6)$ & $3(3)$ & $4(3)$ & $8(5)$ & $3(2)$ & $3(2)$ & $2(1)$ & \\
\hline Moderately & $32(58)$ & $35(48)$ & $47(49)$ & $53(57)$ & 76 (58) & $84(55)$ & $86(59)$ & $87(52)$ & $94(51)$ & \\
\hline Poorly & $6(11)$ & $10(14)$ & $11(12)$ & $10(11)$ & $16(12)$ & $26(17)$ & $23(16)$ & $29(18)$ & $32(18)$ & \\
\hline Unknown & $13(24)$ & $26(36)$ & $32(33)$ & $27(30)$ & $36(27)$ & $36(23)$ & 35 (24) & $47(28)$ & $55(30)$ & \\
\hline PT & & & & & & & & & & 0.620 \\
\hline IDC & $48(87)$ & $68(93)$ & $80(83)$ & $86(93)$ & $119(90)$ & $140(90)$ & $136(93)$ & $153(92)$ & $169(92)$ & \\
\hline ILC & $0(0)$ & $1(1)$ & $1(1)$ & $1(1)$ & $2(2)$ & $2(1)$ & $0(0)$ & $1(1)$ & $2(1)$ & \\
\hline Others & $7(13)$ & $4(6)$ & $15(16)$ & $6(7)$ & $12(8)$ & $12(8)$ & $11(8)$ & $12(7)$ & $12(7)$ & \\
\hline MS & & & & & & & & & & 0.043 \\
\hline $\mathrm{HR}+/ \mathrm{HER} 2-$ & $32(58)$ & $49(67)$ & $51(53)$ & $50(54)$ & $68(52)$ & $90(58)$ & $72(49)$ & $89(54)$ & $93(51)$ & \\
\hline $\mathrm{HR}+/ \mathrm{HER} 2+$ & $9(16)$ & $9(12)$ & $7(7)$ & $5(5)$ & $9(7)$ & $21(14)$ & $15(10)$ & $22(13)$ & $23(13)$ & \\
\hline HR-/HER2+ & $3(6)$ & $6(8)$ & $6(6)$ & $5(5)$ & $12(9)$ & $13(8)$ & $12(8)$ & $11(7)$ & $7(4)$ & \\
\hline HR-/HER2- & $8(15)$ & $6(8)$ & $20(21)$ & $26(28)$ & $33(25)$ & $16(10)$ & $28(19)$ & $27(16)$ & $37(20)$ & \\
\hline Unknown & $3(6)$ & $3(4)$ & $12(13)$ & 7 (8) & $10(8)$ & $14(9)$ & $20(14)$ & $17(10.2)$ & $23(13)$ & \\
\hline Final surgery & & & & & & & & & & 0.792 \\
\hline $\mathrm{BCS}$ & $15(27)$ & $22(30)$ & $23(24)$ & $17(18)$ & $30(23)$ & $32(21)$ & $34(23)$ & $42(25)$ & $42(23)$ & \\
\hline Mastectomy & $40(72)$ & $51(70)$ & $73(76)$ & $76(82)$ & $102(77)$ & $122(79)$ & $113(77)$ & $124(75)$ & $141(77)$ & \\
\hline
\end{tabular}

All data are given as no. of patients (\%). Percentages may not add up to $100 \%$ as a result of rounding

LNM, lymph node metastasis; HG, histological grade; PT, pathological type; IDC, invasive ductal carcinoma; ILC, invasive lobular carcinoma; NACT, neoadjuvant chemotherapy; MS, molecular subtype; BCS, breast conserving surgery

90\%. The 5-year OS for patients with HR+/HER2-, HR+/HER2+, HR-/HER2+, and HR-/HER2- was $94.3 \%$ (95\% CI, $92.0-95.9 \%), \quad 87.3 \%$ (95\% CI, 79.5-92.3\%),
77.9\% (95\% CI, 66.5-85.8\%), and 92.7\% (95\% CI, 88.095.6\%), respectively. The 5-year LRFI and RRFI were highest in patients with the HR+/HER2- subtype $(95.6 \%$ 


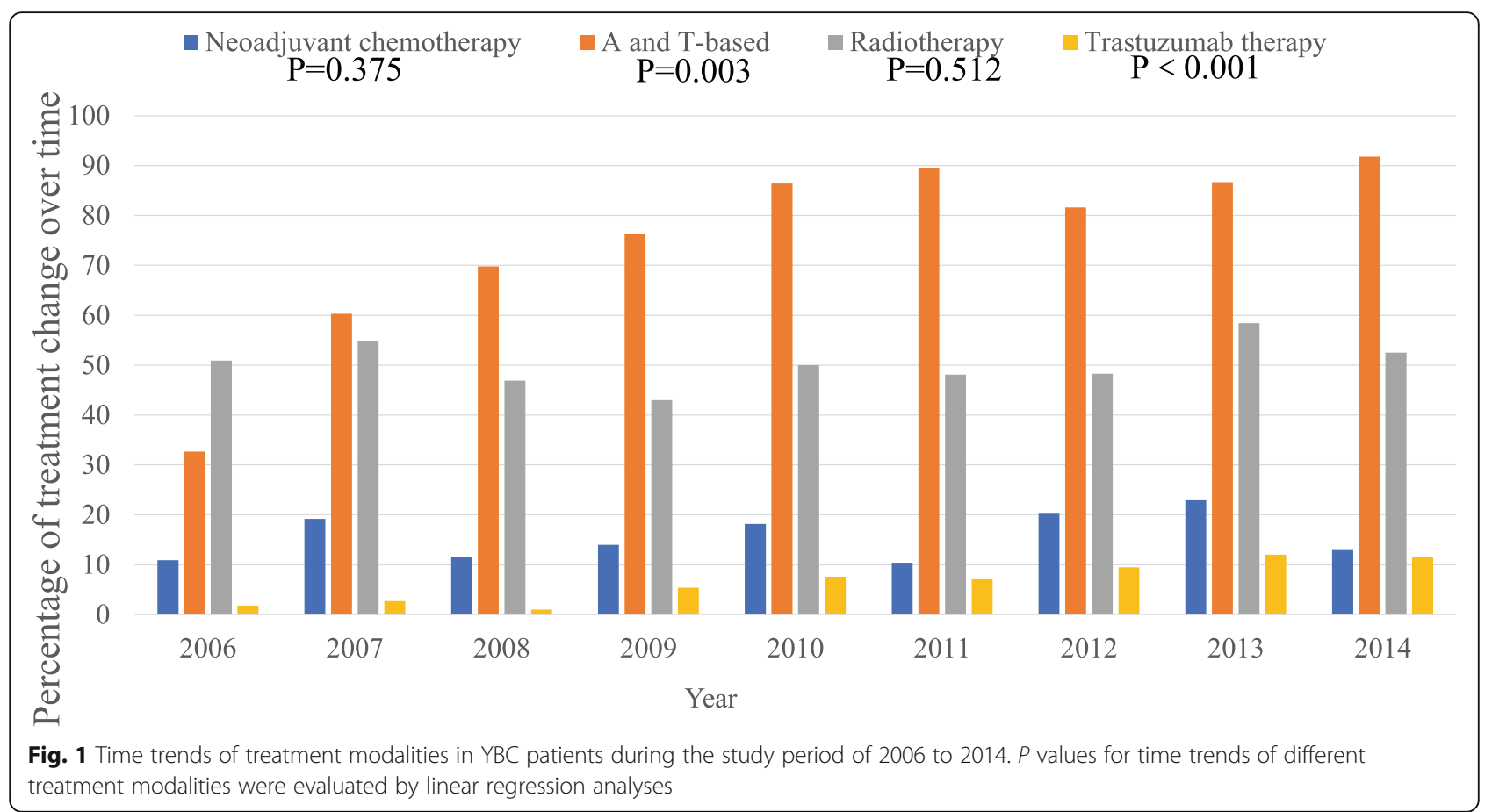

[95\% CI, 94.0-97.0\%] and 95.5\% [95\% CI, 93.4-97.0\%], respectively). For patients with triple-negative tumors, the 10-year DMFI was $>85 \%$, which was higher than the other molecular subtypes. Figure 4 shows the NelsonAalen cumulative hazard rates for LRFI and DMFI by tumor subtype. Patients with HR-/HER2+ status had a significantly higher LR (HR, 20.4; 95\% CI, 11.8-35.4) and DM (HR, 37.2; 95\% CI, 24.6-56.3) at 10 years.

\section{Discussion}

We found a statistically different decreasing trend in the LR and RR rates over time in this large retrospective cohort study of young women with operable invasive breast cancer. This research also revealed that the LR and DM rates varied with the molecular subtype. Tumor size and endocrine therapy were associated with LR, while lymph node metastases and suppression of ovarian function impacted $R R$ based on the multivariate analysis. The 5-year OS of YBC patients was > 90\%, with HR $-/$ HER2 + tumors having the worst survival.

The overall 5-year rates of developing LR, RR, and DM were $6.7 \%, 5.1 \%$, and $16.6 \%$, respectively. Several studies have reported various rates of LRR of YBC patients. LR occurred in $5.4 \%$ of the entire population (7.6\% of those who underwent breast-conserving surgery [BCS] and $2.6 \%$ of those who underwent a mastectomy). An RR of $0.6 \%$ after BCS versus $2.6 \%$ after mastectomy during 11 years of follow-up in women with breast cancer $\leq 35$ of age were collected from the Ontario Cancer Registry between 1994 and 2003 [13]. A study conducted

Table 4 5-year LR, RR, and DM rates of young breast cancer patients treated between 2006 and 2014

\begin{tabular}{|c|c|c|c|c|c|c|c|}
\hline Year & No. of patients & LR, no. (\%) & $P$ & RR, no. (\%) & $P$ & DM, no. (\%) & $P$ \\
\hline Overall & 1099 & $69(6.7)$ & & $52(5.1)$ & & $176(16.6)$ & \\
\hline 2006 & 55 & $7(13.0)$ & 0.028 & $4(7.4)$ & 0.015 & $10(18.2)$ & 0.228 \\
\hline 2007 & 73 & $5(7.3)$ & & $10(14.4)$ & & $14(19.4)$ & \\
\hline 2008 & 96 & $12(13)$ & & $9(9.8)$ & & $17(18.3)$ & \\
\hline 2009 & 93 & $4(4.5)$ & & $3(3.3)$ & & $16(17.2)$ & \\
\hline 2010 & 132 & $7(5.6)$ & & $9(7.1)$ & & $17(13.1)$ & \\
\hline 2011 & 154 & $12(8.2)$ & & $7(4.7)$ & & $32(21.3)$ & \\
\hline 2012 & 147 & $8(6.1)$ & & $2(1.5)$ & & $25(17.6)$ & \\
\hline 2013 & 166 & $8(4.9)$ & & $5(3.2)$ & & $20(12.3)$ & \\
\hline 2014 & 182 & $6(3.4)$ & & $3(1.7)$ & & $25(15.8)$ & \\
\hline
\end{tabular}

$L R$, local recurrence; $R R$, regional recurrence; $D M$, distant metastasis 
a

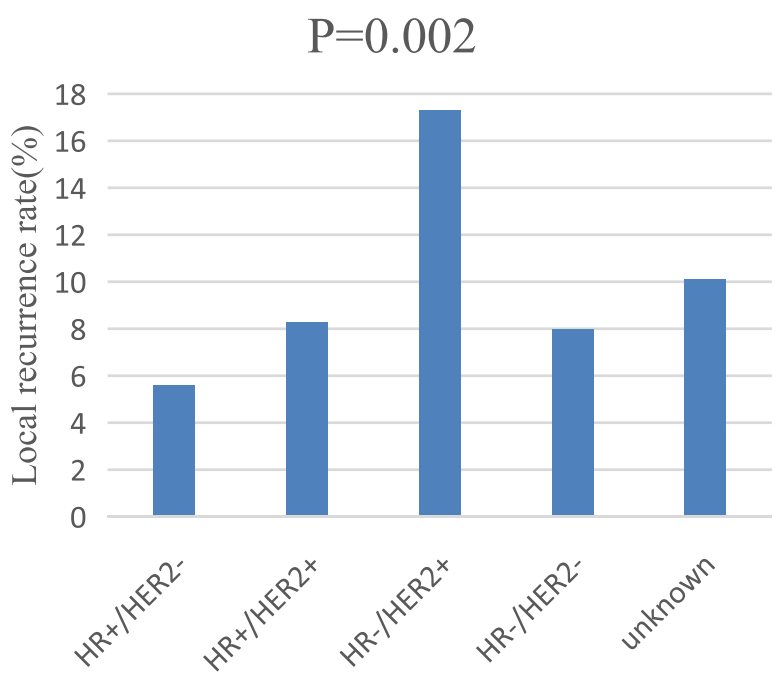

molecular subtype

C

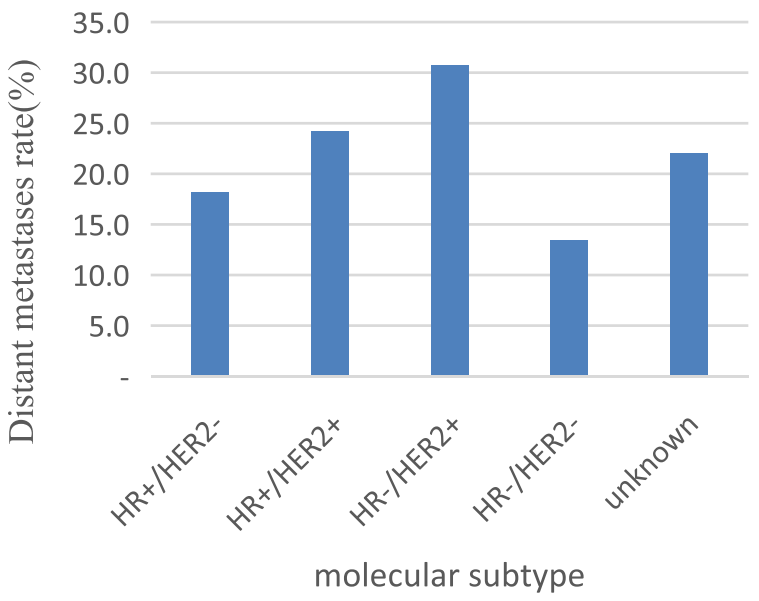

b

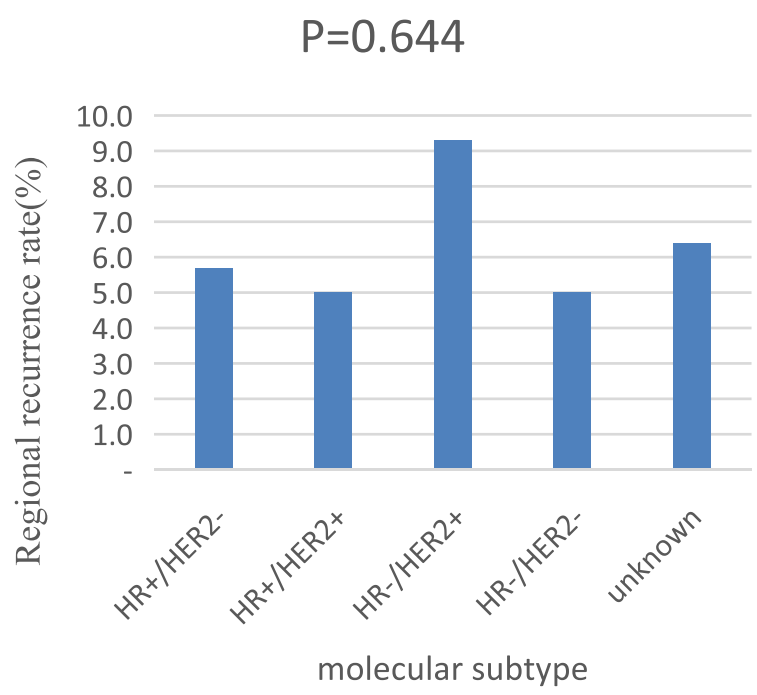

Fig. 2 LR (a), RR (b), and DM (c) rates of young breast cancer patients treated between 2006 and 2014 according to various biomarker subtypes $(n=1099)$

by Aalders et al. reported that young patients $<35$ years of age with early-stage breast cancer had a 5 -year cumulative incidence of $\mathrm{LR}, \mathrm{RR}$, and DM of $3.5 \%, 3.7 \%$, and 13.9\% between 2003 and 2008, respectively [14]. Another study reported a cohort of 3024 patients $18-40$ years of age diagnosed with breast cancer a 5-year LRR rate of $2.63 \%$ after mastectomy versus $5.33 \%$ after BCS (HR, 3.39; 95\% CI, 2.03-5.66; $P<0.001$ ) [12]. The previous studies likely showed lower rates of LRR because earlystage breast cancer accounted for a large proportion of the study subjects. Patients with stages I and II breast cancer made up $75 \%$ of the cohort in our study, while the percentage reached $95 \%$ in the study conducted by Aalders et al. [14].
The rates of LR and RR demonstrated a significant decreasing trend during the period of our study. The results of our research were consistent with previous studies [14-16, 21, 35]. A study conducted by Cossetti et al. divided 7178 patients with biopsy-proven stage IIII breast cancer into cohort 1 (C1) and 2 (C2) who were diagnosed between 1986 and 1992, and mid-2004 and 2008, respectively. The authors demonstrated that the hazard rate of relapse was nearly halved in all yearly intervals to year 9 in $\mathrm{C} 2$ compared with $\mathrm{C} 1$ among the overall population [21]. The patients $<40$ years of age in this research accounted for $13.2 \%$ of patients, and a subsequent study involving patients $<35$ years of age showed overall 5-year rates for LR and RR decreased 
Table 5 Univariate analysis of $L R, R R$, and DM of young breast cancer patients

\begin{tabular}{|c|c|c|c|c|c|c|}
\hline \multirow[t]{2}{*}{ Characteristics } & \multicolumn{2}{|l|}{ LR } & \multirow{2}{*}{$\begin{array}{l}\text { RR } \\
\text { HR }(95 \% \mathrm{Cl})\end{array}$} & \multicolumn{3}{|l|}{ DM } \\
\hline & HR $(95 \% \mathrm{Cl})$ & $P$ & & $P$ & HR $(95 \% \mathrm{Cl})$ & $P$ \\
\hline Tumor size & & $<0.01$ & & $<0.01$ & & $<0.01$ \\
\hline $\mathrm{T} 1$ & 1.0 & & 1.0 & & 1.0 & \\
\hline $\mathrm{T} 2$ & $0.8(0.5-1.3)$ & 0.40 & $0.9(0.5-1.7)$ & 0.85 & $1.9(1.3-2.7)$ & $<0.01$ \\
\hline T3 & $2.2(1.1-4.5)$ & 0.02 & $3.4(1.6-7.0)$ & $<0.01$ & $5.7(3.7-8.7)$ & $<0.01$ \\
\hline $\mathrm{T} 4$ & $8.5(3.3-22.0)$ & $<0.01$ & $9.2(3.1-27.0)$ & $<0.01$ & $13.7(7.1-26.5)$ & $<0.01$ \\
\hline Stage & & $<0.01$ & & $<0.01$ & & $<0.01$ \\
\hline । & 1.0 & & 1.0 & & 1.0 & \\
\hline$\| \mathrm{a}$ & $1.5(0.8-2.9)$ & 0.22 & $0.4(0.2-1.1)$ & 0.09 & $2.1(1.2-3.5)$ & $<0.01$ \\
\hline$\| \mathrm{b}$ & $2.0(1.0-4.3)$ & 0.06 & $2.0(0.9-4.5)$ & 0.08 & $3.4(1.9-6.0)$ & $<0.01$ \\
\hline III a & $1.0(0.4-2.7)$ & 0.92 & $3.6(1.7-7.5)$ & $<0.01$ & $6.2(3.6-10.6)$ & $<0.01$ \\
\hline III b & $7.8(1.8-34.3)$ & $<0.01$ & $4.3(0.6-33.1)$ & 0.16 & $14.8(5.5-39.6)$ & $<0.01$ \\
\hline III C & $3.8(1.5-9.5)$ & $<0.01$ & $4.1(1.8-9.1)$ & $<0.01$ & $12.9(7.6-21.8)$ & $<0.01$ \\
\hline LN metastasis & & $<0.01$ & & $<0.01$ & & $<0.01$ \\
\hline No & 1.0 & & 1.0 & & 1.0 & \\
\hline N1 & $2.3(1.4-3.7)$ & $<0.01$ & $2.3(1.1-4.6)$ & 0.02 & $2.1(1.5-3.0)$ & $<0.01$ \\
\hline $\mathrm{N} 2$ & $1.0(0.4-2.3)$ & 0.95 & $5.9(3.0-11.5)$ & $<0.01$ & $3.8(2.6-5.6)$ & $<0.01$ \\
\hline N3 & $3.9(2.1-7.3)$ & $<0.01$ & $6.9(3.3-14.5)$ & $<0.01$ & $8.1(5.6-11.8)$ & $<0.01$ \\
\hline $\mathrm{HG}$ & & 0.09 & & 0.81 & & 0.21 \\
\hline I & 1.0 & & 1.0 & & 1.0 & \\
\hline$\|$ & $0.6(0.2-1.6)$ & 0.26 & $0.7(0.2-2.4)$ & 0.60 & $1.9(0.7-5.3)$ & 0.19 \\
\hline III & $1.1(0.4-3.3)$ & 0.86 & $1.0(0.3-3.5)$ & 0.97 & $2.5(0.9-7.0)$ & 0.08 \\
\hline Final surgery & & 0.13 & & 0.30 & & $<0.01$ \\
\hline Mastectomy & 1.0 & & 1.0 & & 1.0 & \\
\hline $\mathrm{BCS}$ & $1.4(0.9-2.3)$ & 0.13 & $0.7(0.4-1.3)$ & 0.30 & $0.4(0.3-0.7)$ & $<0.01$ \\
\hline ER status & & $<0.01$ & & 0.16 & & 0.35 \\
\hline Positive & 1.0 & & 1.0 & & 1.0 & \\
\hline Negative & $2.0(1.3-3.1)$ & $<0.01$ & $1.6(1.0-2.7)$ & 0.06 & $1.2(0.9-1.6)$ & 0.25 \\
\hline PR status & & $<0.01$ & & 0.94 & & 0.93 \\
\hline Positive & 1.0 & & 1.0 & & 1.0 & \\
\hline Negative & $1.9(1.2-2.9)$ & $<0.01$ & $1.1(0.7-1.9)$ & 0.72 & $1.1(0.8-1.4)$ & 0.71 \\
\hline HER2 status & & $<0.01$ & & 0.59 & & $<0.01$ \\
\hline Positive & 1.0 & & 1.0 & & 1.0 & \\
\hline Negative & $0.5(0.3-0.8)$ & $<0.01$ & $0.8(0.4-1.4)$ & 0.38 & $0.6(0.4-0.8)$ & $<0.01$ \\
\hline MS & & $<0.01$ & & 0.66 & & $<0.01$ \\
\hline HR+/HER2- & 1.0 & & 1.0 & & 1.0 & \\
\hline HR+/HER2+ & $1.7(0.8-3.4)$ & 0.15 & $1.0(0.4-2.3)$ & 0.94 & $1.5(1.0-2.2)$ & 0.06 \\
\hline HR-/HER2+ & $3.5(1.8-6.7)$ & $<0.01$ & $1.7(0.8-3.9)$ & 0.18 & $1.9(1.2-2.9)$ & $<0.01$ \\
\hline HR-/HER2- & $1.5(0.8-2.7)$ & 0.20 & $0.9(0.4-1.8)$ & 0.73 & $0.7(0.5-1.1)$ & 0.15 \\
\hline NACT & & 0.04 & & $<0.01$ & & $<0.01$ \\
\hline No & 1.0 & & 1.0 & & 1.0 & \\
\hline Yes & $1.8(1.0-3.0)$ & 0.04 & $2.5(1.4-4.3)$ & $<0.01$ & $2.9(2.1-3.8)$ & $<0.01$ \\
\hline $\mathrm{ACT}$ & & 0.18 & & 0.70 & & 0.29 \\
\hline A- and T-based & 1.0 & & 1.0 & & 1.0 & \\
\hline
\end{tabular}


Table 5 Univariate analysis of LR, RR, and DM of young breast cancer patients (Continued)

\begin{tabular}{|c|c|c|c|c|c|c|}
\hline \multirow[t]{2}{*}{ Characteristics } & \multicolumn{2}{|l|}{ LR } & \multirow{2}{*}{$\begin{array}{l}\text { RR } \\
\text { HR }(95 \% \mathrm{Cl})\end{array}$} & \multicolumn{3}{|l|}{ DM } \\
\hline & HR $(95 \% \mathrm{Cl})$ & $P$ & & $P$ & HR $(95 \% \mathrm{Cl})$ & $P$ \\
\hline A-based & $1.2(0.7-2.2)$ & 0.47 & $1.3(0.7-2.5)$ & 0.48 & $1.2(0.8-1.7)$ & 0.42 \\
\hline Radiotherapy & & 0.20 & & 0.03 & & $<0.01$ \\
\hline No & 1.0 & & 1.0 & & 1.0 & \\
\hline Yes & $1.1(0.7-1.8)$ & 0.56 & $1.9(1.1-3.3)$ & 0.03 & $2.5(1.8-3.5)$ & $<0.01$ \\
\hline ET & & $<0.01$ & & 0.09 & & 0.22 \\
\hline No & 1.0 & & 1.0 & & 1.0 & \\
\hline Yes & $0.5(0.3-0.7)$ & $<0.01$ & $0.7(0.4-1.3)$ & 0.30 & $1.1(0.8-1.5)$ & 0.71 \\
\hline OFS & & 0.52 & & $<0.01$ & & $<0.01$ \\
\hline No & 1.0 & & 1.0 & & 1.0 & \\
\hline Yes & $1.2(0.6-2.1)$ & 0.64 & $3.4(1.8-6.2)$ & $<0.01$ & $3.7(2.6-5.3)$ & $<0.01$ \\
\hline$\pi$ & & 0.30 & & 0.94 & & 0.14 \\
\hline No & 1.0 & & 1.0 & & 1.0 & \\
\hline Yes & $1.5(0.7-3.4)$ & 0.30 & $0.9(0.3-2.6)$ & 0.94 & $1.4(0.9-2.3)$ & 0.14 \\
\hline
\end{tabular}

over time [14]. We studied the time trend of tumor characteristics and treatment modalities and per incidence year of patients. It revealed that the proportion of stages I and II breast cancer increased, while stage III showed a downward trend over the 9 years. The proportion of patients received with anthracycline- and taxane-based chemotherapy regimens and treated with trastuzumab increased during the study time. These findings might explain, in part, the decreasing trend of $L R$ and RR rates over time.

We observed a downtrend in the recurrence of DM over time, although the difference was not statistically significant. Previous studies have reported similar results $[14,17,36]$. Therefore, we suggest that the improvement in OS among patients with breast cancer is closely associated with the lower DM rates in recent years [16, 19, 37, 38].

Our study reported BCS did not significantly affect LRR of young patients with breast cancer. In previous studies, BCS was associated with an increased risk of LR in young patients $[39,40]$. Owing to a significant advancement in the treatment of breast ancer, current studies showed BCS had no significant increase in risk of recurrence compared with mastectomy [15, 41].

Patients with HR-/HER2+ tumors (HER2 overexpressing tumors) had the highest LR rates, while $\mathrm{HR}+$ / HER2 - tumors (luminal tumors) displayed the lowest LR rates among the entire cohort. A systematic review identifying patients from 15 studies appraised the effect of molecular subtype on LRR according to the type of surgery and the authors suggested patients with triplenegative and HER2 over-expressing subtypes were at high risk of developing LRR, and luminal tumors exhibited the lowest LRR rates [25], which was in agreement with our findings. A cohort of 394 early-stage invasive breast cancer patients undergoing BCS were classified as luminal A, luminal B, HER-2, and basal phenotype. The reported crude LRR rates of the basal phenotype were highest (17.3\%), followed by HER-2 (15.4\%), luminal B (8.7\%), and luminal A (5\%) [24]. A five-biomarker panel (ER, PR, HRE-2, CK5/6, and EGFR) was used to categorize the tumors, which is not a commonly intrinsic molecular phenotype of breast cancer, and therefore it is not useful clinically. However, the results of our research differed slightly from those of published studies $[14,26,42]$. These studies reported no difference in LR among patients with various tumor subtypes. We found that molecular subtype was a prognostic factor for both LR and DM, but not an independent prognostic factor for LR based on the Cox proportional hazard model.

We found the cumulative probability of 5- and 10-year OS was $91.9 \%$ and $86.2 \%$, respectively, in YBC patients $\leq$ 35 years of age in our study. A population-based study of women diagnosed with breast cancer from 1992 to 2005 demonstrated that the breast cancer-specific survival of patients $<35$ years of age was $69 \%$ at the 10 -year follow-up evaluation [27]. Miller et al. reported that the 5 -year breast cancer net survival in females diagnosed between 2001 and 2009 was $88.2 \%$ independent of race and age, and the survival rates improved from 2001 and 2003 to 2004 and 2009 [38]. Another study suggested that the 5-year breast cancer-specific survival increased from $74.0 \%$ during $1975-1979$ to $88.5 \%$ during 2010 2015 in women diagnosed between ages 20 and 39 years from the SEER database [20]. The data obtained in our research were slightly higher than previous studies, which might be due to the recent study year accompanied 
Table 6 Multivariate analysis of LR, RR, and DM of young breast cancer patients

\begin{tabular}{|c|c|c|c|c|c|c|}
\hline \multirow[t]{2}{*}{ Characteristics } & \multicolumn{2}{|l|}{ LR } & \multirow{2}{*}{$\begin{array}{l}\text { RR } \\
\text { HR }(95 \% \mathrm{Cl})\end{array}$} & \multicolumn{3}{|l|}{ DM } \\
\hline & HR $(95 \% \mathrm{Cl})$ & $P$ & & $P$ & HR $(95 \% \mathrm{Cl})$ & $P$ \\
\hline Tumor size & & $<0.01$ & & 0.11 & & 0.01 \\
\hline $\mathrm{T} 1$ & 1.0 & & 1.0 & & 1.0 & \\
\hline $\mathrm{T} 2$ & $0.5(0.2-1.0)$ & 0.05 & $0.6(0.3-1.4)$ & 0.24 & $1.4(0.9-2.3)$ & 0.16 \\
\hline T3 & $1.7(0.6-4.8)$ & 0.33 & $1.2(0.5-2.9)$ & 0.74 & $1.8(1.0-3.2)$ & 0.04 \\
\hline $\mathrm{T} 4$ & $6.5(1.2-36.9)$ & 0.03 & $4.2(1.0-17.2)$ & 0.05 & $5.4(2.0-14.0)$ & $<0.01$ \\
\hline Stage & & 0.24 & & 0.09 & & 0.57 \\
\hline । & 1.0 & & 1.0 & & 1.0 & \\
\hline$\| \mathrm{a}$ & $1.7(0.7-4.2)$ & 0.22 & $0.5(0.2-1.6)$ & 0.26 & $1.4(0.7-2.8)$ & 0.28 \\
\hline$\| \mathrm{b}$ & $2.1(0.6-7.8)$ & 0.25 & $2.2(0.6-8.8)$ & 0.25 & $1.3(0.5-3.4)$ & 0.53 \\
\hline III a & $0.7(0.2-3.5)$ & 0.69 & $0.9(0.2-4.4)$ & 0.90 & $2.1(0.8-5.8)$ & 0.15 \\
\hline III b & $0.9(0.1-9.0)$ & 0.96 & $0.3(0.0-4.8)$ & 0.43 & $1.2(0.3-4.9)$ & 0.81 \\
\hline III C & $0.5(0.1-3.6)$ & 0.53 & $0.2(0.0-1.5)$ & 0.11 & $4.2(1.0-17.8)$ & 0.05 \\
\hline LN metastasis & & 0.06 & & 0.02 & & 0.59 \\
\hline No & 1.0 & & 1.0 & & 1.0 & \\
\hline N1 & $2.0(1.0-4.0)$ & 0.05 & $1.6(0.6-4.1)$ & 0.32 & $1.5(0.9-2.6)$ & 0.14 \\
\hline N2 & $1.01(0.3-3.5)$ & 0.93 & $7.1(1.7-29.2)$ & $<0.01$ & $1.3(0.6-2.9)$ & 0.50 \\
\hline N3 & $6.9(1.4-35.0)$ & 0.02 & $34.7(4.0-304.1)$ & $<0.01$ & $1.3(0.3-4.7)$ & 0.73 \\
\hline Final surgery & & - & & - & & 0.14 \\
\hline Mastectomy & - & & - & & 1.0 & \\
\hline $\mathrm{BCS}$ & - & - & - & - & $0.7(0.4-1.1)$ & 0.14 \\
\hline ER status & & 0.70 & & - & & - \\
\hline Positive & 1.0 & & - & & - & \\
\hline Negative & $1.5(0.6-3.9)$ & 0.40 & - & - & - & - \\
\hline PR status & & 0.54 & & - & & - \\
\hline Positive & 1.0 & & - & & - & \\
\hline Negative & $1.5(0.6-3.6)$ & 0.41 & - & - & - & - \\
\hline HER2 status & & 0.92 & & - & & - \\
\hline Positive & 1.0 & & - & & - & \\
\hline Negative & $0.0(0.0-\mathrm{NA})$ & 0.92 & - & - & - & - \\
\hline MS & & 0.49 & & - & & 0.01 \\
\hline HR+/HER2- & 1.0 & & - & & 1.0 & \\
\hline HR+/HER2+ & $0.0(0.0-\mathrm{NA})$ & 0.92 & - & - & $1.4(0.9-2.1)$ & 0.15 \\
\hline HR-/HER2+ & $0.0(0.0-\mathrm{NA})$ & 0.92 & - & - & $2.5(1.5-4.4)$ & $<0.01$ \\
\hline HR-/HER2- & $0.3(0.1-1.2)$ & 0.10 & - & - & $1.9(1.1-3.2)$ & 0.02 \\
\hline NACT & & 0.57 & & 0.11 & & 0.04 \\
\hline No & 1.0 & & 1.0 & & 1.0 & \\
\hline Yes & $1.2(0.7-2.2)$ & 0.57 & $1.6(0.9-3.0)$ & 0.11 & $1.4(1.0-2.0)$ & 0.04 \\
\hline Radiotherapy & & - & & 0.10 & & 0.64 \\
\hline No & - & & 1.0 & & 1.0 & \\
\hline Yes & - & - & $0.5(0.2-1.1)$ & 0.09 & $1.2(0.7-2.0)$ & 0.41 \\
\hline ET & & 0.07 & & - & & - \\
\hline No & 1.0 & & - & & - & \\
\hline Yes & $0.4(0.2-1.0)$ & 0.04 & - & - & - & - \\
\hline
\end{tabular}


Table 6 Multivariate analysis of LR, RR, and DM of young breast cancer patients (Continued)

\begin{tabular}{|c|c|c|c|c|c|c|}
\hline \multirow[t]{2}{*}{ Characteristics } & \multicolumn{2}{|l|}{ LR } & \multirow{2}{*}{$\begin{array}{l}\text { RR } \\
\text { HR }(95 \% \mathrm{Cl})\end{array}$} & \multicolumn{3}{|l|}{ DM } \\
\hline & HR $(95 \% \mathrm{Cl})$ & $P$ & & $P$ & HR (95\% Cl) & $P$ \\
\hline OFS & & - & & 0.03 & & $<0.01$ \\
\hline No & - & & 1.0 & & 1.0 & \\
\hline Yes & - & - & $2.4(1.2-4.6)$ & $<0.01$ & $3.7(2.3-5.8)$ & $<0.01$ \\
\hline
\end{tabular}

$U A$, univariate analysis; $M A$, multivariate analysis; $H G$, histological grade; $M S$, molecular subtype; $N A C T$, neoadjuvant chemotherapy; $A C T$, adjuvant chemotherapy;

$E T$, endocrine therapy; $\pi$, trastuzumab therapy; $N A$, not arrived

by the improved treatment methods. In addition, the 10 years of follow-up data were not available for patients between 2010 and 2014. Lastly, our study might be limited by the single-center and retrospective nature. In short, the survival rate of $\mathrm{YBC}$ patients has improved in recent years.

The characteristics and treatment of $\mathrm{BC}$ appeared to be different between young and old women. Mustacchi et.al reported $85.5 \%$ of patients aged $\geq 65$ years had at least one positive receptor while the proportion in our cohort was $64.9 \%$. The use of chemotherapy (especially taxane regimen) was significantly decreasing with age in old patients [43]. However, almost all the young patients received chemotherapy after surgery and the use of taxanes increased over time in our study. The 5-year rates of LR were higher in young patients (6.7\%) than patients $\geq 50$ years $(3.7 \%)$, whereas the 5 -year OS $(91.9 \%)$ was comparable with OS reported for older patients (91.0\%) [44]. Possible explanations are that younger patients might be treated more aggressively after LR and have fewer comorbidities and other diseases than older patients.

Our findings demonstrated that the differences in prognosis among YBC patients varied the with molecular subtype. Women with HR-/HER2+ had the worst LRFI, RRFI, DMFI, and OS compared with the other subtypes, which was consistent with previous articles [24, 29, 30,33]. Nevertheless, many studies have indicated that YBC patients with luminal B subtype had a worse prognosis $[27,28,31,32]$. The reason causing the discrepant results might be connected to the year of the study (i.e., there was no HER2-targeted therapy until 1998). After the development of HER2-targeted therapy, the survival of HER2-positive patients was greatly improved [45]. We found HR-/HER2+ had the worst prognosis in our study. It was slightly inconsistent with the current view that triple-negative breast cancer had the worst prognosis. The reasons might be that patients with HR-/HER2+ statuses had larger tumors and more

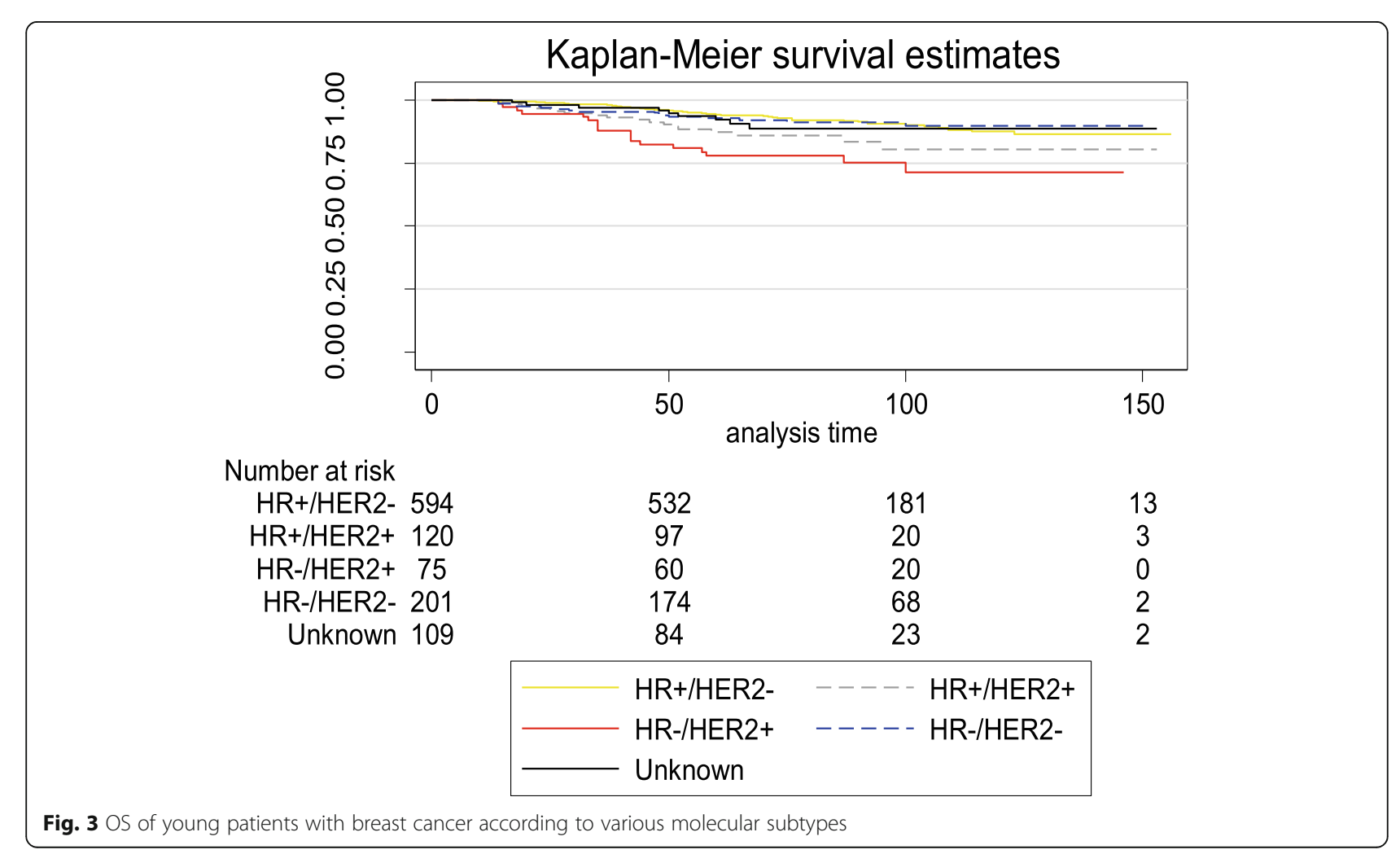


Table 7 Relapse and survival of outcomes in young breast cancer patients according to various molecular subtypes

\begin{tabular}{|c|c|c|c|c|c|c|}
\hline \multirow[t]{2}{*}{ Outcome } & \multicolumn{3}{|l|}{ 5-year } & \multicolumn{3}{|l|}{ 10-year } \\
\hline & 5-year estimate (\%) & $95 \% \mathrm{Cl}$ & Total number of events & 10-year estimate (\%) & $95 \% \mathrm{Cl}$ & Total number of events \\
\hline \multicolumn{7}{|l|}{ Overall } \\
\hline LRFI & 93.3 & 91.6 to 94.7 & 61 & 90.4 & 87.9 to 92.5 & 71 \\
\hline RRFI & 95.0 & 93.4 to 96.1 & 47 & 92.4 & 90.1 to 94.2 & 57 \\
\hline DMFI & 83.5 & 81.1 to 85.6 & 155 & 78.3 & 75.4 to 80.9 & 186 \\
\hline OS & 91.9 & 90.1 to 93.4 & 76 & 86.2 & 83.2 to 88.7 & 101 \\
\hline \multicolumn{7}{|l|}{ HR+/HER- } \\
\hline LRFI & 95.6 & 94.0 to 97.0 & 25 & 92.7 & 89.0 to 95.1 & 32 \\
\hline RRFI & 95.5 & 93.4 to 97.0 & 25 & 91.9 & 88.3 to 94.5 & 34 \\
\hline DMFI & 85.6 & 82.4 to 88.2 & 83 & 78.5 & 74.4 to 82.1 & 107 \\
\hline OS & 94.3 & 92.0 to 95.9 & 32 & 87.6 & 83.2 to 91.0 & 49 \\
\hline \multicolumn{7}{|l|}{$\mathrm{HR}+/ \mathrm{HER}+$} \\
\hline LRFI & 90.7 & 83.4 to 94.9 & 10 & 90.7 & 83.4 to 94.9 & 10 \\
\hline RRFI & 94.5 & 88.0 to 97.5 & 6 & 94.5 & 88.1 to 97.5 & 6 \\
\hline DMFI & 77.1 & 68.1 to 83.8 & 26 & 73.4 & 63.9 to 80.8 & 29 \\
\hline OS & 87.3 & 79.5 to 92.3 & 14 & 80.3 & 68.1 to 88.3 & 17 \\
\hline \multicolumn{7}{|l|}{ HR-/HER+ } \\
\hline LRFI & 83.1 & 72.0 to 90.0 & 12 & 81.3 & 69.9 to 88.8 & 13 \\
\hline RRFI & 91.8 & 82.7 to 96.3 & 6 & 90.0 & 80.0 to 95.1 & 7 \\
\hline DMFI & 71.7 & 59.9 to 80.5 & 20 & 68.6 & 56.6 to 77.9 & 23 \\
\hline OS & 77.9 & 66.5 to 85.8 & 16 & 71.4 & 56.8 to 81.8 & 18 \\
\hline \multicolumn{7}{|l|}{ HR-/HER2- } \\
\hline LRFI & 92.5 & 87.6 to 95.5 & 14 & 90.6 & 84.7 to 94.3 & 16 \\
\hline RRFI & 94.7 & 90.4 to 97.1 & 10 & 94.7 & 90.4 to 97.1 & 10 \\
\hline DMFI & 86.7 & 81.1 to 90.8 & 26 & 86.1 & 80.3 to 90.2 & 27 \\
\hline OS & 92.7 & 88.0 to 95.6 & 14 & 89.9 & 83.8 to 93.8 & 17 \\
\hline
\end{tabular}

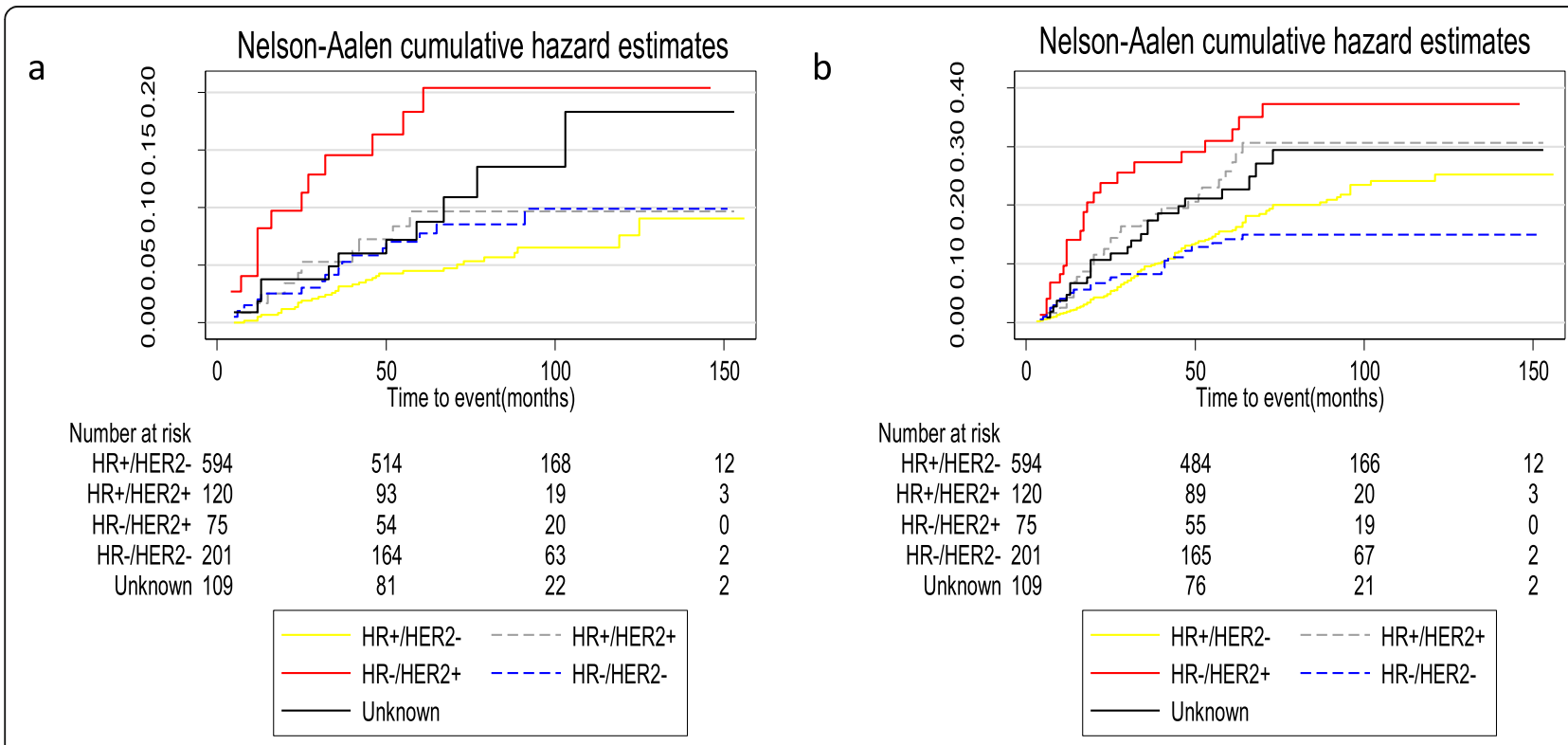

Fig. 4 Nelson-Aalen cumulative hazard estimates for LRFI (a) and DMFI (b) for all young patients by molecular subtypes 
lymph node metastases. In addition, inferior treatment might be another reason causing poor prognosis. Only 83 cases received trastuzumab therapy of the 194 HRE2-positive patients in our study. With the rapid development of HER2-targeted therapies, such as the combination of trastuzumab and pertuzumab, and neratinib and T-DM1, the outcomes of HER2-positive patients could be further improved [46-48].

However, there were some limitations in our study. First, molecular subtypes were categorized according to HR and HER2 status without other marks, such as Ki67, and analyses of HER2 status were limited by FISH testing that was not performed in some cases. Thus, we could not further subdivide the molecular subtypes. Second, information concerning adherence to adjuvant endocrine therapy and ovary function suppression, such as goserelin, was not available on medical records obtained through the subsequent follow-up. Therefore, the reliability of information might be affected by recall bias. Third, the median follow-up of 82 months was relatively short for YBC patients. Finally, the patients were collected in a large single center in northern China and is not population based. As a result, the experiences of patients in our study might not be generalizable to all young women with breast cancer.

\section{Conclusions}

In conclusion, the overall 5-year LR and RR rates with YBC patients were low and showed a decreasing trend and the proportion of early-stage breast cancer increased between 2006 and 2014. The highest LR rates in this young population were associated with HR-/HER2+ tumors. We expect to develop more new treatments to prolong the survival time and improve the quality of life of young women with breast cancer in the near future.

\section{Abbreviations \\ LR: Local recurrenceRRRegional recurrence; LRR: Loco-regional recurrence; YBC: Young breast cancer; HR: Hormone receptor; HER2: Human epidermal growth factor receptor 2; OS: Overall survival; DM: Distant metastasis; LRFI: Local recurrence-free interval; DMFI: Distant metastasis-free interval; RRFI: Regional recurrence-free interval; ER: Estrogen receptor}

\section{Authors' contributions}

$Y L$ designed the study, performed the research, and wrote the paper. $Y Z$ performed research and analyzed data. SL performed research and checked the data. YD helped performed research and checked the data. HL designed the study and analyzed data. The author(s) read and approved the final manuscript.

\section{Funding}

This research was supported by Tianjin Major Scientific and Technological Special Project for Major Disease Prevention and Control [19ZXDBSY00090].

\section{Availability of data and materials}

The datasets used during the present study are available from the corresponding author on reasonable request.

\section{Declarations}

\section{Ethics approval and consent to participate}

All procedures performed in studies involving human participants were in accordance with the ethical standards of the Institutional Research

Committee and with the 1964 Helsinki Declaration and its later amendments or comparable ethical standards. This study was approved by the Ethics Committee of Tianjin Medical University Cancer Institution and Hospital (Approval No. bc2021001). The need for informed patient consent was waived because of the retrospective nature of the study. As a retrospective study, a waiver of informed consent was approved by the Ethics Committee of Tianjin Medical University Cancer Institution and Hospital.

\section{Consent for publication}

Not applicable

\section{Competing interests}

The authors declare that they have no competing interests.

\section{Author details}

${ }^{1}$ The Second Surgical Department of Breast Cancer, Tianjin Medical University Cancer Institute and Hospital, National Clinical Research Center for Cancer \& Key Laboratory of Cancer Prevention and Therapy, Tianjin's Clinical Research Center for Cancer, Tianjin 300060, China. ${ }^{2}$ Oncology Department, Taikang Xianlin Drum Tower Hospital, School of Medicine, Nanjing University, Nanjing 210046, Jiangsu Province, China. ${ }^{3}$ Oncology Department, Hebei PetroChina Central Hospital, Langfang 065000, Hebei Province, China.

Received: 18 August 2020 Accepted: 26 March 2021

Published online: 13 April 2021

\section{References}

1. DeSantis CE, Ma J, Goding Sauer A, Newman LA, Jemal A. Breast cancer statistics, 2017, racial disparity in mortality by state. CA Cancer J Clin. 2017; 67(6):439-48. https://doi.org/10.3322/caac.21412.

2. Siegel RL, Miller KD, Jemal A. Cancer statistics, 2019. CA Cancer J Clin. 2019; 69(1):7-34. https://doi.org/10.3322/caac.21551.

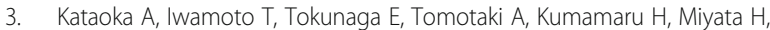
et al. Young adult breast cancer patients have a poor prognosis independent of prognostic clinicopathological factors: a study from the Japanese Breast Cancer Registry. Breast Cancer Res Tr. 2016;160(1):163-72. https://doi.org/10.1007/s10549-016-3984-8.

4. Nixon AJ, Neuberg D, Hayes DF, Gelman R, Connolly JL, Schnitt S, et al. Relationship of patient age to pathologic features of the tumor and prognosis for patients with stage I or II breast cancer. J Clin Oncol. 1994; 12(5):888-94. https://doi.org/10.1200/JCO.1994.12.5.888.

5. Anders CK, Hsu DS, Broadwater G, Acharya CR, Foekens JA, Zhang Y, et al. Young age at diagnosis correlates with worse prognosis and defines a subset of breast cancers with shared patterns of gene expression. J Clin Oncol. 2008;26(20):3324-30. https://doi.org/10.1200/Jco.2007.14.2471.

6. Azim HA, Michiels S, Bedard PL, Singhal SK, Criscitiello C, Ignatiadis M, et al. Elucidating prognosis and biology of breast cancer arising in young women using gene expression profiling. Clin Cancer Res. 2012;18(5):1341-51. https:// doi.org/10.1158/1078-0432.Ccr-11-2599.

7. Tichy JR, Lim E, Anders CK. Breast cancer in adolescents and young adults: a review with a focus on biology. J Natl Compr Canc Ne. 2013;11(9):1060-9. https://doi.org/10.6004/jnccn.2013.0128.

8. Anders CK, Fan C, Parker JS, Carey LA, Blackwell KL, Klauber-DeMore N, et al. Breast carcinomas arising at a young age: unique biology or a surrogate for aggressive intrinsic subtypes? J Clin Oncol. 2011;29(1):E18-20. https://doi. org/10.1200/Jco.2010.28.9199

9. Azim HA, Nguyen B, Brohee S, Zoppoli G, Sotiriou C: Genomic aberrations in young and elderly breast cancer patients. BMC Med 2015, 13.doi: ARTN 26610.1186/s12916-015-0504-3.

10. Honrado E, Benitez J, Palacios J. The molecular pathology of hereditary breast cancer: genetic testing and therapeutic implications. Modern Pathol. 2005;18(10):1305-20. https://doi.org/10.1038/modpathol.3800453.

11. Bollet MA, Sigal-Zafrani B, Mazeau V, Savignoni A, de la Rochefordiere A, Vincent-Salomon A, et al. Age remains the first prognostic factor for locoregional breast cancer recurrence in young $(<40$ years) women treated with 
breast conserving surgery first. Radiother Oncol. 2007;82(3):272-80. https:// doi.org/10.1016/j.radonc.2007.01.001.

12. Maishman T, Cutress Rl, Hernandez A, Gerty S, Copson ER, Durcan L, et al. Local Recurrence and breast oncological surgery in young women with breast cancer: the POSH observational cohort study. Ann Surg. 2017;266(1): 165-72. https://doi.org/10.1097/SLA.0000000000001930.

13. Quan ML, Paszat LF, Fernandes K, Sutradhar R, McCready DR, Rakovitch E, et al. The effect of surgery type on survival and recurrence in very young women with breast cancer. J Clin Oncol. 2013;31(15).

14. Aalders KC, Postma EL, Strobbe LJ, van der Heiden-van der Loo M, Sonke GS, Boersma $\sqcup$, et al. Contemporary locoregional recurrence rates in young patients with early-stage breast cancer. J Clin Oncol. 2016;34(18):2107. https://doi.org/10.1200/Jco.2015.64.3536.

15. van Laar C, van der Sangen MJC, Poortmans PMP, Nieuwenhuijzen GAP, Roukema JA, Roumen RMH, et al. Local recurrence following breastconserving treatment in women aged 40 years or younger: trends in risk and the impact on prognosis in a population-based cohort of 1143 patients. Eur J Cancer. 2013;49(15):3093-101. https://doi.org/10.1016/j.ejca.2 013.05.030.

16. Botteri E, Veronesi P, Vila J, Rotmensz N, Galimberti V, Thomazini MV, et al. Improved prognosis of young patients with breast cancer undergoing breast-conserving surgery. Brit J Surg. 2017;104(13):1802-10. https://doi. org/10.1002/bjs.10658.

17. Yerushalmi R, Woods R, Kennecke H, Speers C, Knowling M, Gelmon K. Patterns of relapse in breast cancer: changes over time. Breast Cancer Res Treat. 2010;120(3):753-9. https://doi.org/10.1007/s10549-009-0510-2.

18. Malmgren JA, Mayer M, Atwood MK, Kaplan HG. Differential presentation and survival of de novo and recurrent metastatic breast cancer over time: 1990-2010. Breast Cancer Res Treat. 2018;167(2):579-90. https://doi.org/10.1 007/s10549-017-4529-5.

19. Jensen MB, Ejlertsen B, Mouridsen HT, Christiansen P, Grp DBCC. Improvements in breast cancer survival between 1995 and 2012 in Denmark: the importance of earlier diagnosis and adjuvant treatment. Acta Oncol. 2016;55(sup2):24-35. https://doi.org/10.3109/0284186x.2015.1128119.

20. Guo F, Kuo Y-F, Shih YCT, Giordano SH, Berenson AB. Trends in breast cancer mortality by stage at diagnosis among young women in the United States. Cancer. 2018;124(17):3500-9. https://doi.org/10.1002/cncr.31638.

21. Cossetti RJD, Tyldesley SK, Speers CH, Zheng YN, Gelmon KA. Comparison of breast cancer recurrence and outcome patterns between patients treated from 1986 to 1992 and from 2004 to 2008. J Clin Oncol. 2015;33(1):65-U106. https://doi.org/10.1200/Jco.2014.57.2461.

22. Park S, Koo JS, Kim MS, Park HS, Lee JS, Lee JS, et al. Characteristics and outcomes according to molecular subtypes of breast cancer as classified by a panel of four biomarkers using immunohistochemistry. Breast. 2012;21(1): 50-7. https://doi.org/10.1016/j.breast.2011.07.008.

23. Partridge AH, Hughes ME, Warner ET, Ottesen RA, Wong Y-N, Edge SB, et al. Subtype-Dependent relationship between young age at diagnosis and breast cancer survival. Journal of Clinical Oncology. 2016;34(27):3308-14. https://doi.org/10.1200/JCO.2015.65.8013.

24. Millar EKA, Graham PH, O'Toole SA, McNeil CM, Browne L, Morey AL, et al. Prediction of local recurrence, distant metastases, and death after breastconserving therapy in early-stage invasive breast cancer using a fivebiomarker panel. J Clin Oncol. 2009;27(28):4701-8. https://doi.org/10.1200/ Jco.2008.21.7075.

25. Lowery AJ, Kell MR, Glynn RW, Kerin MJ, Sweeney KJ. Locoregional recurrence after breast cancer surgery: a systematic review by receptor phenotype. Breast Cancer Res Treat. 2012;133(3):831-41. https://doi.org/10.1 007/s10549-011-1891-6.

26. Lai SF, Chen YH, Kuo WH, Lien HC, Wang MY, Lu YS, et al. Locoregional recurrence risk for postmastectomy breast cancer patients with T1-2 and one to three positive lymph nodes receiving modern systemic treatment without radiotherapy. Ann Surg Oncol. 2016;23(12):3860-9. https://doi.org/1 0.1245/s10434-016-5435-5.

27. Fredholm H, Magnusson K, Lindstrom LS, Garmo H, Falt SE, Lindman H, et al. Long-term outcome in young women with breast cancer: a population-based study. Breast Cancer Res Treat. 2016;160(1):131-43. https://doi.org/10.1007/s10549-016-3983-9.

28. Tang LC, Jin $X$, Yang HY, He M, Chang $H$, Shao ZM, et al. Luminal $B$ subtype: a key factor for the worse prognosis of young breast cancer patients in China. BMC Cancer. 2015;15(1):201. https://doi.org/10.1186/ s12885-015-1207-z.
29. Carey LA, Perou CM, Livasy CA, Dressler LG, Cowan D, Conway K, et al. Race, breast cancer subtypes, and survival in the Carolina Breast Cancer Study. JAMA. 2006;295(21):2492-502. https://doi.org/10.1001/jama.295.21.2492.

30. Keegan THM, Press DJ, Tao L, DeRouen MC, Kurian AW, Clarke CA, Gomez SL: Impact of breast cancer subtypes on 3-year survival among adolescent and young adult women. Breast Cancer Res 2013, 15(5).doi:ARTN R9510. $1186 / \mathrm{bcr} 3556$

31. Cancello G, Maisonneuve P, Rotmensz N, Viale G, Mastropasqua MG, Pruneri $\mathrm{G}$, et al. Prognosis and adjuvant treatment effects in selected breast cancer subtypes of very young women ( $<35$ years) with operable breast cancer. Ann Oncol. 2010;21(10):1974-81. https://doi.org/10.1093/annonc/mdq072.

32. Villarreal-Garza C, Mohar A, Bargallo-Rocha JE, Lasa-Gonsebatt F, ReynosoNoveron N, Matus-Santos J, et al. Molecular Subtypes and Prognosis in Young Mexican Women With Breast Cancer. Clin Breast Cancer. 2017;17(3): e95-e102. https://doi.org/10.1016/j.clbc.2016.11.007.

33. Ignatov A, Eggemann $H$, Burger $E$, Ignatov T. Patterns of breast cancer relapse in accordance to biological subtype. J Cancer Res Clin Oncol. 2018; 144(7):1347-55. https://doi.org/10.1007/s00432-018-2644-2.

34. Hammond ME, Hayes DF, Dowsett M, Allred DC, Hagerty KL, Badve S, et al. American Society of Clinical Oncology/College Of American Pathologists guideline recommendations for immunohistochemical testing of estrogen and progesterone receptors in breast cancer. J Clin Oncol. 2010;28(16): 2784-95. https://doi.org/10.1200/JCO.2009.25.6529.

35. Saphner T, Tormey DC, Gray R. Annual hazard rates of recurrence for breast cancer after primary therapy. J Clin Oncol. 1996;14(10):2738-46. https://doi. org/10.1200/JCO.1996.14.10.2738.

36. Colleoni M, Sun Z, Price KN, Karlsson P, Forbes JF, Thurlimann B, et al. Annual hazard rates of recurrence for breast cancer during 24 years of follow-up: results from the International Breast Cancer Study Group trials I to V. J Clin Oncol. 2016;34(9):927-35. https://doi.org/10.1200/JCO.2015.62.3504.

37. Imkampe AK, Bates T. Improvements in breast cancer survival over time, related to adjuvant treatment and node status. Eur J Surg Oncol. 2009;35(2): 151-5. https://doi.org/10.1016/j.ejso.2008.02.010.

38. Miller JW, Smith JL, Ryerson AB, Tucker TC, Allemani C: Disparities in breast cancer survival in the United States (2001-2009): findings from the CONCORD-2 study. Cancer 2017, 123 Suppl 24(Suppl 24):5100-5118.doi: https://doi.org/10.1002/cncr.30988.

39. van der Sangen MJC, van de Wiel FMM, Poortmans PMP, Tjan-Heijnen VCG, Nieuwenhuijzen GAP, Roumen RMH, et al. Voogd AC: are breast conservation and mastectomy equally effective in the treatment of young women with early breast cancer? Long-term results of a population-based cohort of 1,451 patients aged $\leq 40$ years. Breast Cancer Res Tr. 2011;127(1): 207-15. https://doi.org/10.1007/s10549-010-1110-X.

40. Voogd AC, Nielsen M, Peterse JL, Blichert-Toft M, Bartelink H, Overgaard M, et al. Differences in risk factors for local and distant recurrence after breastconserving therapy or mastectomy for stage I and II Breast cancer: pooled results of two large european randomized trials. J Clin Oncol. 2001;19(6): 1688-97. https://doi.org/10.1200/JCO.2001.19.6.1688.

41. Wang LZ, He YJ, Li JF, Wang TF, Xie YT, Fan ZQ, et al. Comparisons of breast conserving therapy versus mastectomy in young and old women with early-stage breast cancer: long-term results using propensity score adjustment method. Breast Cancer Res Tr. 2020;183(3):717-28. https://doi. org/10.1007/s10549-020-05821-0.

42. Haffty BG, Yang QF, Reiss M, Kearney T, Higgins SA, Weidhaas J, et al. Locoregional relapse and distant metastasis in conservatively managed triple negative early-stage breast cancer. J Clin Oncol. 2006;24(36):5652-7. https://doi.org/10.1200/Jco.2006.06.5664.

43. Mustacchi G, Cazzaniga ME, Pronzato P, De Matteis A, Di Costanzo F, Floriani I, et al. Breast cancer in elderly women: a different reality? Results from the NORA study. Ann Oncol. 2007;18(6):991-6. https://doi.org/10.1 093/annonc/mdm063.

44. Courdi A, Doyen J, Gal J, Chamorey E. Local recurrence after breast cancer affects specific survival differently according to patient age. Oncology. 2010; 79(5-6):349-54. https://doi.org/10.1159/000323483.

45. Cameron D, Piccart-Gebhart MJ, Gelber RD, Procter M, Goldhirsch A, de

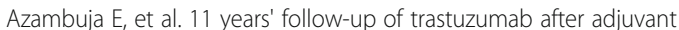
chemotherapy in HER2-positive early breast cancer: final analysis of the HERceptin Adjuvant (HERA) trial. Lancet. 2017;389(10075):1195-205. https:// doi.org/10.1016/S0140-6736(16)32616-2.

46. von Minckwitz G, Procter M, de Azambuja E, Zardavas D, Benyunes M, Viale G, et al. Adjuvant Pertuzumab and Trastuzumab in Early HER2-Positive 
Breast Cancer. New Engl J Med. 2017;377(2):122-31. https://doi.org/10.1056/ NEJMoa1703643.

47. Park JW, Liu MC, Yee D, Yau C, van 't Veer LJ, Symmans WF, et al. Adaptive randomization of neratinib in early breast cancer. New Engl J Med. 2016; 375(1):11-22. https://doi.org/10.1056/NEJMoa1513750.

48. T-DM1 Extends Survival in HER2+ Breast Cancer. Cancer Discov. 2016;6(2): OF4. https://doi.org/10.1158/2159-8290.CD-NB2015-174.

\section{Publisher's Note}

Springer Nature remains neutral with regard to jurisdictional claims in published maps and institutional affiliations.

Ready to submit your research? Choose BMC and benefit from:

- fast, convenient online submission

- thorough peer review by experienced researchers in your field

- rapid publication on acceptance

- support for research data, including large and complex data types

- gold Open Access which fosters wider collaboration and increased citations

- maximum visibility for your research: over $100 \mathrm{M}$ website views per year

At BMC, research is always in progress.

Learn more biomedcentral.com/submissions 